\title{
Konzept einer vorausschauenden Kommunikation in der palliativen Behandlung von Patienten mit pneumologisch-onkologischen Erkrankungen
}

\author{
Concept for a Forward-Looking Communication in the Palliative Treatment \\ of Patients with Pneumological-Oncological Diseases Prospective
}

Autoren

Institut
G. Tessmer, O. Zaba, C. Grohé

Evangelische Lungenklinik Berlin, Klinik für Pneumologie (Prof. Dr. med. C. Grohé) eingereicht 17.11.2011 akzeptiert nach Revision 21.2. 2011

\section{Bibliografie}

Dol http://dx.doi.org/ 10.1055/s-0030-1256307

Online-Publikation: 24. 3. 2011

Pneumologie 2011; 65:

503-509 @ Georg Thieme

Verlag KG Stuttgart · New York ISSN 0934-8387

Korrespondenzadresse Dipl..Psych. Günter Tessmer Evangelische Lungenklinik Berlin Lindenberger Weg 27

13125 Berlin

guenter.tessmer@elk-berlin.de

\section{Zusammenfassung \\ $\nabla$}

Die Betreuung von Patienten mit Lungenkarzinomen ist in einer Vielzahl der Fälle geprägt durch die Kürze der verbleibenden Lebenszeit. Die zeitliche Bedrängnis kann zu erheblichen Problemen im Umgang mit Patienten, Angehörigen und Pflegenden führen. Ein Konzept der partnerschaftlichen Perspektiventwicklung über standardisierte Kommunikationsschritte könnte einen Weg weisen in eine behutsamere zwischenmenschliche Begegnung. Gelingt die Umsetzung dieser Strategie, so ist ein erheblicher Zugewinn an Lebensqualität für die Patienten und Angehörigen und an Arbeitspraktikabilität für die behandelnden Ärzte zu erwarten.

\section{Einleitung}

\section{$\nabla$}

Thorakale Tumore, insbesondere das Lungenkarzinom, zeichnen sich in $70 \%$ der Fälle durch eine Inkurabilität bei Diagnosestellung aus. Trotz erheblicher diagnostischer Bemühungen in der jüngeren Vergangenheit hat sich dieser Prozentsatz bis heute nicht wesentlich verändert [1]. Im Regelfall bedeutet die Diagnosestellung für diese Patienten ein Ausblick auf eine limitierte Lebenszeit mit palliativ ausgerichteten Behandlungsangeboten [2]. Die palliativen Behandlungen erstrecken sich über Wochen, Monate und Jahresfrist hinaus. Sie untergliedern sich in die palliative tumororientierte Behandlung wie zum Beispiel die palliative Chemotherapie inklusive supportiver Begleitbehandlung und die palliativmedizinische Symptombehandlung. Die Länge der Krankenhausaufenthalte ist für die Patienten und ihre Angehörigen schlecht kalkulierbar. Immer wieder können unvorhergesehene Behandlungskomplikationen die klinischen und damit auch die privaten Organisationspläne durcheinanderbringen. Sowohl für die Patienten und Angehörigen als auch für die behandelnden Ärzte ist der Umgang

\section{Abstract \\ $\nabla$}

The treatment and medical care of patients with lung cancer is mostly characterised and complicated by a reduced life expectancy. The lack of time can easily be the cause for various problems concerning the handling of these patients, their relatives and medical staff. A concept for opening future perspectives through standardised steps of communication could lead to a more cautious and humane relationship between physician and patient. In the case of successful realisation of this strategy, a significant enhancement of quality of life for the patient and relatives and practicability of work for the physician can be expected.

mit den wechselhaften Widrigkeiten rund um die Krankheit und deren Behandlung psychisch äußerst belastend [3]. Prospektive Analysen, die untersuchen, inwieweit durch partnerschaftliche Kommunikationsmodelle Patienten und Angehörige besser unterstützt werden, liegen bis heute nur in ungenügender Form vor. Es zeichnet sich jedoch in letzter Zeit ab, dass eine vorsorgend vorausschauende Kommunikation und eine mit der palliativen Tumorbehandlung von Anfang an verbundene palliativmedizinische Symptombehandlung die Prognose bei Patienten mit fortgeschrittenem Lungenkarzinom erheblich verbessern [4]. Basierend auf den Arbeitserfahrungen der letzten Jahre im Umgang mit Lungenkarzinompatienten in unserer Fachklinik ist das folgende Konzept entwickelt worden. Ziel ist, die psychischen Belastungen der Patienten, ihrer Angehörigen und der behandelnden Ärzte durch ein Modell vorsorgend vorausschauender Kommunikationsschritte zu reduzieren. In Ergänzung zum täglichen vertrauensvollen Arzt-Patient-Kontakt soll die Möglichkeit akzentuiert werden, gemeinsam mit den Patienten und ihren Angehörigen beiseite zu treten, Informationen ordnend nachzustrukturieren, 
Verständnis sichernd zu bündeln und in ihrem wesentlichen Bedeutungsgehalt für die nächsten Behandlungsschritte auf den Punkt zu bringen.

Es soll erreicht werden, dass die Ärzte an vorhersehbaren Wendepunkten der Behandlung bewusst innehalten und im Abgleich mit den Bedürfnisäußerungen der Patienten und Angehörigen inhaltlich und methodisch in Revision gehen, eine Standortbestimmung vornehmen, wechselseitiges Einvernehmen bekräftigen und in geteilter Verantwortung fortfahren mit der Behandlung.

Es soll erreicht werden, dass die Patienten, ihre Angehörigen und die behandelnden Ärzte Schritt für Schritt die dissenten Sichtweisen auf die Krankheit und ihre Behandlung minimieren und die konsenten maximieren.

Es geht in diesem Artikel mitnichten darum, ein geschlossenes Entwicklungsresultat vorzustellen. Die Entwicklungsarbeit ist noch auf dem Wege und wird es wohl auch stets bleiben. Es geht vielmehr darum, den aktuellen Entwicklungsstand einer kritischen Betrachtung zuzuführen und eine konstruktive Diskussion anzuregen, die weiterhilft in dem täglichen Bestreben, gerade auch Patienten mit inkurablen Erkrankungen die bestmögliche Unterstützung zukommen zu lassen.

\section{Inhaltliche und methodische Bezugspunkte}

$\nabla$

\section{Begriffsdefinition: Kommunikation versus Gesprächsführung}

In der Folge wird der Begriff Kommunikation anstelle des Begriffs Gesprächsführung benutzt. Der Begriff Gesprächsführung impliziert streng genommen ein Interaktionsgefälle. Es gibt jemanden, der oben steht, und jemanden, der unten steht. Gesprächsführungskonzeptionen weisen so gesehen nicht in eine Interaktion auf Augenhöhe. Es stellen sich unter anderem Fragen wie:

- Wer führt wen, wer folgt wem?

- Woher weiß derjenige, der führen soll, wohin er führen soll?

- Weiß derjenige, der folgen soll, wohin er folgen soll?

- Will derjenige, der folgen soll, aus freien Stücken dahin folgen, wohin er geführt werden soll?

Der Begriff Kommunikation liegt uns näher, weil er eine Interaktionssymmetrie impliziert. Er entstammt etymologisch dem lateinischen Verb „communicare“ mit den Kernbedeutungen: etwas miteinander teilen, etwas einander mitteilen. Kommunikationskonzepte, die von dieser Kernbedeutung ausgehen, weisen in eine partnerschaftlich-gleichberechtigte Interaktion.

Es geht uns nicht darum, ein eingangs bestehendes Interaktionsgefälle zwischen einem kranken Menschen, der sich schwach und hilfsbedürftig erlebt, und einem Arzt, der fachkompetent Hilfe anbietet, in Abrede zu stellen und damit den Hilfesuchenden heillos zu überfordern. Es geht uns viel mehr darum, die Kommunikation zwischen Patienten, ihren Angehörigen und den behandelnden Ärzten inhaltlich und methodisch darauf auszurichten, dass sich im Laufe der Behandlung das Eingangsgefälle Schritt für Schritt verringern kann.

\section{Begriffsdefinition Palliativmedizin WHO 2002:}

"Palliativmedizin ist ein Ansatz zur Verbesserung der Lebensqualität von Patienten und ihren Familien, die mit den Problemen konfrontiert sind, die mit einer lebensbedrohlichen Erkrankung einhergehen, und zwar durch Vorbeugen und Lindern von Leiden, durch frühzeitiges Erkennen, untadelige Einschätzung und
Behandlung von Schmerzen und anderen Beschwerden körperlicher, psychosozialer und spiritueller Art."

Die WHO definiert 2002 die Palliativmedizin als einen Behandlungsansatz, der auf eine Verbesserung der Lebensqualität abzielt bei Patienten und ihren Angehörigen, die mit den drängenden Problemen rund um eine inkurable Erkrankung konfrontiert sind. Ihren Leiden soll vorausschauend und vorsorgend begegnet werden. Sie sollen möglichst frühzeitig erkannt und bedürfnisgerecht gelindert werden [5]. Damit wird im Vergleich zu früheren Definitionen ein Paradigmenwechsel vollzogen. Nicht mehr das Krankheitsstadium, sondern die psychophysische Bedürftigkeit der Patienten indiziert den Einsatz einer rein symptomatischen Behandlung. Sofern die psychophysische Bedürftigkeit der Patienten bereits sehr früh in der palliativen Tumorbehandlung gegeben ist, möglicherweise schon nach wenigen Wochen, rückt die palliativmedizinische Symptombehandlung ganz nach vorn in der Behandlungschronologie. Eine sensible, bedürfnisgerechte Rehabilitation im Sinne der Ermöglichung eines selbstbestimmten Lebens innerhalb der jeweiligen erkrankungsbedingten Grenzen wird zum Kernziel der Palliativmedizin.

\section{Begriffsdefinition Lebensqualität}

Die Patienten bewerten ihre Lebensqualität höchst individuell am Maßstab ihres subjektiven Befindens. Viele Versuche, die Lebensqualität objektiv mit Scores zu beschreiben, erreichen bisher eher Annäherungen an eine objektive Beurteilbarkeit [6]. Als Destillat aus den an der Oberfläche variierenden individuellen Lebensqualitätsbewertungen lässt sich unseres Erachtens ein überindividueller Definitionszusammenhang herausstellen:

Das Erleben von Lebensqualität scheint von einem Handlungsfähigkeitserleben in Bezug auf unmittelbare und mittelbare Problemlagen abhängig zu sein. Je handlungsfähiger die Patienten und Angehörigen sich erleben, desto besser fühlen sie sich, je handlungsunfähiger sie sich erleben, desto schlechter fühlen sie sich. Selbiges scheint auch auf ihre Ärzte zuzutreffen.

\section{Vorsorgevollmacht}

Angesichts der gegenwärtigen Gesetzeslage im Hinblick auf das rechtssichere Umgehen mit nichteinwilligungsfähigen Patienten scheint es uns ratsam, vorausschauend Sorge zu tragen, dass die Patienten über einen elektiv eingesetzten Vorsorgebevollmächtigten ihre Entscheidungsfähigkeit dauerhaft sichern für Krankheits- und Behandlungssituationen, in denen sie nicht einsichtsund erklärungsfähig sind.

Beunruhigt durch die strittige öffentliche Debatte in den Medien um Patientenverfügung und Vorsorgevollmacht wenden sich mittlerweile viele Patienten und Angehörige an unsere Ärzte mit ihren Fragen zu diesem Themenkreis. Sie äußern ihre Sorge, in Krankheitssituationen zu geraten, in denen sie sich nicht selbst verständlich machen können und über ihren Kopf hinweg mit Behandlungen belegt werden, die sie aus innerster Überzeugung ablehnen.

Die behandelnden Ärzte sind ihrerseits gehalten, für das Fortbestehen ihrer rechtssicheren Handlungsfähigkeit vorausschauend Sorge zu tragen. Sie dürfen das kommunikative Gegenüber nicht verlieren, bleiben sie doch stets auf die konsente Zusammenarbeit mit dem Entscheidungsträger Patient verwiesen [7].

Bereits bei der Aufnahme in unser Krankenhaus wird jedem Patienten als Teil seiner Informationsmappe ein Informationsschreiben zur Vorsorgevollmacht und ein Standardformular für die Gesundheitssorge zur Kenntnisnahme ausgehändigt. Gemäß unserer justiziarischen Abstimmung ist das Formular entlastend 
für die Patienten und Angehörigen auf drei Festlegungen gekürzt worden:

- Mit welchen Personen dürfen die behandelnden Ärzte über die Krankheit und deren Behandlung sprechen?

- Welche Personen dürfen im Auftrag der Patienten Verhandlungen führen und rechtsverbindlich unterschreiben?

- Welche Personen dürfen im Auftrag der Patienten über die Durchführung von Behandlungen entscheiden?

Um dem misslichen Umstand entgegenzuwirken, dass Patienten und Angehörige sich in der Auseinandersetzung mit dem Informationsschreiben zur Vorsorgevollmacht alleingelassen und überbeansprucht fühlen könnten, bietet unser Sozialdienst wunschgemäß eine vertiefende persönliche Beratung an und leistet wunschgemäß praktische Hilfestellung beim Ausfüllen des Standardformulars.

Eine bestätigende Unterschrift eines Zeugen oder eine notarielle Beglaubigung sind mit Blick auf die rechtliche Verbindlichkeit nicht nötig. Die Bevollmächtigung gilt nur für den Zeitraum der Einsichts- und Erklärungsunfähigkeit der Patienten. Ist diese wieder gegeben, erlischt die Bevollmächtigung augenblicklich ohne expliziten Widerruf.

Bei der Erstdiagnose der Lungentumorerkrankung (Stadium IIIB/ IV), unmittelbar vor Einleitung der palliativen tumororientierten Behandlung, fragt in unserem Hause der aufklärende Arzt nach dem Bestehen einer Patientenverfügung oder Vorsorgevollmacht. Bezug nehmend auf das Informationsschreiben zur Vorsorgevollmacht erläutert er den Patienten und ihren Angehörigen die Grundintention dieses Handlungsvorschlags. Die Patienten und Angehörigen empfinden unsere Vorgehensweise als in ihrem Sinne hilfreich und praktikabel. Viele entschließen sich vorausschauend dazu, eine Vorsorgevollmacht zur Gesundheitssorge in unserer gekürzten Form bereits zu diesem Zeitpunkt abzufassen. Im Laufe der Behandlung entscheiden die Stationsärzte in einem Selbsteinschätzung und Fremdeinschätzung abgleichenden Gespräch mit den Patienten darüber, ob ihre Einsichts- und Erklärungsfähigkeit gegeben ist oder nicht. Sollten Uneinigkeiten oder Zweifel bestehen, kann zur letzten Beurteilung ein Psychiater konsiliarisch hinzugezogen werden.

Die Stationsärzte tragen mitverantwortlich Sorge dafür, dass die Vorsorgebevollmächtigten im Laufe des vorausschauenden Kommunikationsprozesses stets entscheidungsfähig bleiben. In der Situation tatsächlicher Entscheidungsübernahme erhalten die Vorsorgebevollmächtigten eine ausführliche, verständliche Aufklärung im Hinblick auf die Krankheitssituation, in der sie im Auftrag des Patienten über den Behandlungsvorschlag entscheiden sollen.

\section{Die operationale Handlungsorientierung an der}

\section{Handlungsleitlinie der geteilten Verantwortung}

Die Patienten und ihre Angehörigen werden gemäß dieser Handlungsleitlinie ausdrücklich zum Mitdenken und mitverantwortlichen Handeln eingeladen (Einladungsstruktur). Das theoretische und praktische Fachwissen der behandelnden Ärzte und das Erfahrungswissen der Patienten und Angehörigen soll zu einem soliden gemeinsamen Wissen um die gemeinsam zu tragende Sache verschränkt werden (kooperative Expertise). Vor- und Nachteile möglicher Behandlungsentscheidungen werden partnerschaftlich abgewogen. Ein Behandlungskonsens (informed consent) wird erarbeitet und in geteilter Verantwortlichkeit umgesetzt. Diese partnerschaftliche Handlungsorientierung schließt ein, dass als Ergebnis des Aufklärungsprozesses auch eine Ent- scheidung der Patienten gegen die vorgeschlagene Behandlung erster Wahl ärztlicherseits mitgetragen wird.

\section{Die vorausschauende Kommunikation $\nabla$}

Die tumororientierte palliative Behandlung wie zum Beispiel die palliative Chemotherapie sollte unseres Erachtens in Ergänzung zum täglichen vertrauensvollen Arzt - Patientkontakt von Beginn an mit einem System vorausschauender kommunikativer Handlungsschritte verbunden werden. Sowohl die Patienten und Angehörigen als auch die behandelnden Ärzte können so entlang des wechselvollen Krankheits- und Behandlungsverlaufs Verständnis sicherndes Hintergrund- und Zusammenhangswissen entwickeln, das sie in der symptomatischen palliativen Behandlung reibungsärmer und zielgenauer interagieren lässt.

Die folgenden standardisierten Kommunikationsformen erscheinen auf den ersten Blick inhaltlich und methodisch aufwendig, haben sich jedoch in der täglichen klinischen Praxis - wider Erwarten - als äußerst zeitökonomisch erwiesen. Dafür gibt es Erklärungen:

- Die Patienten und ihre Angehörigen lernen auch die Aspekte ihres Krankheits- und Behandlungsverlaufs deutlicher wahrzunehmen und in ihrer Wirkdimension einzuschätzen, die sie, weil ungeliebt, immer wieder aus ihrem Bewusstsein drängen wollen. Sie verstehen die innere Kausalität ihrer Krankheit und deren Behandlung im Laufe des Kommunikationsprozesses immer besser. Sie bekommen den roten Behandlungsfaden schneller wieder zu fassen, sofern sie ihn verloren haben. Redundante Nachfragen und Konflikt behaftete Missverständnisse nehmen deutlich ab.

- Die Patienten und Angehörigen klagen weniger über unkontrollierbar einschießende Angstzustände, innere Unruhe, Gedankenkreisen und daraus resultierende Schlafschwierigkeiten, die zuweilen medikamentöse Interventionshandlungen erfordern können. Sie beschreiben sich auf Nachfrage als psychisch weniger angespannt und als weniger dyspnoeisch. Sie erleben sich physisch belastbarer im Untersuchungs- und Behandlungsalltag und beim nachmittäglichen Spaziergang mit ihren Angehörigen.

- Ärztliche Kollegen bewerten die Systematik der vorausschauenden Kommunikation - gerade auch das Prinzip der wechselseitig abgestimmten, vorausschauenden Gesprächsterminierung - als spürbare Druckentlastung im Stationsalltag. Im Wissen um die gemeinsame Gesprächsvereinbarung mit dem behandelnden Arzt stellen die Patienten und ihre Angehörigen ihre Fragen zur Krankheits- und Behandlungssituation bis dahin zurück. Da sie es mehrfach erfahren haben, verlassen sie sich darauf, dass sie in dem vereinbarten Gespräch ausführliche und verständliche Antworten erhalten und mitsteuernd Einfluss nehmen können auf den Behandlungsprozess.

\section{Kommunikative Handlungsschritte der}

vorausschauenden Kommunikation

Entlang des klinischen Behandlungsverlaufs werden vier kommunikative Handlungsschritte unterschieden:

1. das interdisziplinäre Aufklärungsgespräch bei Diagnosestellung

2. das Perspektivgespräch

3. das Konsensgespräch in der dissenten Behandlungssituation (optional)

4. das Konsensgespräch in der finalen Krankheitssituation 
Die jeweiligen Gesprächsteilnehmer, namentlich der behandelnde Arzt, der Psychoonkologe, die Mitarbeiterin der Bezugspflege (optional), die Physiotherapeutin (optional), die Mitarbeiterin des Sozialdienstes (optional) sind den Patienten und ihren Angehörigen in der Regel aus ihrem Stationsalltag heraus bereits vertraut.

\section{Das interdisziplinäre Aufklärungsgespräch bei Diagnosestellung}

\section{Bei dieser Gesprächsform sollten teilnehmen:}

der Patient und ein Angehöriger seines Vertrauens (Vorsorgebevollmächtigter), der behandelnde Stationsarzt, der Psychoonkologe.

Die Angehörigen werden mit Erlaubnis der Patienten ausdrücklich zum interdisziplinären Aufklärungsgespräch eingeladen. Die Patienten werden so wunschgemäß von der Last befreit, komplexe Informationen weitervermitteln zu müssen. In der Vergangenheit ist es nach unserer Erfahrung manches Mal zu Informationslücken auf Seiten der Angehörigen gekommen, die zuweilen zu konfliktträchtigen Fehleinschätzungen der Krankheitssituation führten. Die Angehörigen werden im interdisziplinären Aufklärungsgespräch demzufolge mit guten Gründen ermuntert, ihre eigenen Fragen zu stellen, ihre eigenen Ängste und Bedenken zu äußern. Es wird auf diese Weise eine hohe Aufklärungs- und Verständnissymmetrie zwischen den Patienten und ihren Angehörigen erzielt.

Mit der psychoonkologischen Beratung kommen die Patienten und ihre Angehörigen wunschgemäß oder auf Empfehlung des für sie zuständigen Arztes in der Regel schon während des ihrerseits Angst besetzten diagnostischen Prozesses in Berührung. Nicht wenige Patienten und Angehörige fragen sodann in eigener Initiative, ob der Psychoonkologe an ihrem Aufklärungsgespräch teilnehmen kann. Zumeist soll er sie dabei unterstützen, dass sie die Ängste und Bedenken, die sie im Vorfeld mit ihm erörtert haben, hinreichend zur Sprache bringen und ohne Scham in ihrem Sprachduktus die Fragen stellen, die sie im Innersten beschäftigen.

\section{Grundthema:}

Das Grundthema des interdisziplinären Aufklärungsgesprächs ist die initiale Aufklärung der Patienten und ihrer Angehörigen über die Krankheit und ihre Behandlungsmöglichkeiten. Es handelt sich um Tumorerkrankungen der Lunge des Stadiums IIIB oder IV, die grundlegend palliativ behandelt werden. Die palliative Tumortherapie, beispielsweise die palliative Chemotherapie im Stadium IIIB/IV, ist (laut S3-Leitlinie Lungenkarzinom) darauf ausgerichtet, den Tumor zu verkleinern, die Tumor bedingten Symptome zu reduzieren, das Voranschreiten der Erkrankung temporär zu begrenzen, letztlich Stillstandssituationen zu erreichen, die den Patienten therapiefreie Zeiten eröffnen und die Überlebenszeit verlängern [8].

Davon zu unterscheiden ist die palliativmedizinische Behandlung, die als rein symptomatische Behandlung ausschließlich darauf ausgerichtet ist, die quälenden Symptome der Grunderkrankung zu lindern und so weit als möglich zu kontrollieren.

Eine symptomatische Behandlung begleitet die palliative Tumorbehandlung stets in Form der supportiven Therapie. Als explizit palliativmedizinische Behandlung tritt sie an die Stelle der palliativen Tumorbehandlung, sofern im Behandlungsverlauf die Stabilisierung des Allgemeinzustands der Patienten medizinisch die oberste Indikationspriorität gewinnt [9].
Es ist anzuraten, den Patienten und ihren Angehörigen die Behandlung des Lungenkarzinoms von Beginn an als aus zwei gleichberechtigten Behandlungssäulen bestehend zu erläutern, aus der tumororientierten Behandlung und der symptomatischen Behandlung.

Die behandelnden Ärzte wissen, dass die Schnittstelle zwischen tumororientierter Behandlung und palliativmedizinischer Symptombehandlung im Behandlungsverlauf definitiv erreicht wird. Sie können schon zu diesem frühen Zeitpunkt den Patienten und ihren Angehörigen die Möglichkeit eröffnen, sich mit einer auf Symptomkontrolle und Stabilisierung des Allgemeinzustands zentrierten Behandlungsform vertraut zu machen, die im Laufe der Tumorbehandlung immer wieder mit guten Gründen gemeinsam in den Vordergrund gestellt werden kann. Die palliativmedizinische Symptombehandlung verliert so ihren schlechten Leumund: Sie wird nicht erst dann ins Spiel gebracht, wenn die tumororientierte Behandlung nicht mehr mit einer umkehrenden Wirkerwartung belegt werden kann oder die Patienten aufgrund ihrer verminderten Allgemeinverfassung gar nicht mehr imstande sind, eine tumororientierte Behandlung auszuhalten [10].

Die palliativmedizinische Symptombehandlung wird unsererseits entsprechend der rehabilitativen Definitionsausrichtung der WHO eingesetzt, zum Beispiel, um über die Stabilisierung der Allgemeinverfassung der Patienten die Handlungsmöglichkeit einer weiteren tumororientierten Behandlung zu öffnen. Patienten und Angehörige, die sich eingeladen fühlen, mitdenkend zu intervenieren, fragen ihrerseits nicht selten, ob eine palliativmedizinische Symptombehandlung in der gerade aktuellen Krankheits- und Behandlungssituation nicht die Behandlung erster Wahl wäre.

Es erscheint uns nicht nur rechtlich unumgänglich, sondern auch in jeglicher Hinsicht erstrebenswert, möglichst alle Behandlungsentscheidungen in kooperativer Expertise mit den Patienten und ihren Angehörigen zu treffen. Sowohl die erwünschten Entwicklungen der Behandlung, zum Beispiel hin zu einer Tumorremission, als auch die unerwünschten, zum Beispiel hin zu einem Tumorprogress, werden so einvernehmlich verantwortet und getragen.

\section{Das Perspektivgespräch}

\section{Bei dieser Gesprächsform sollten teilnehmen:}

der Patient und ein Angehöriger seines Vertrauens (Vorsorgebevollmächtigter), der behandelnde Stationsarzt, der Psychoonkologe, die behandelnde Mitarbeiterin der Bezugspflege (optional). Grundthema:

Die Patienten stehen vor einer einschneidenden Behandlungszäsur: In der palliativen chemotherapeutischen Behandlung wird zum Beispiel nach anfänglichen Remissionsbefunden ein Tumorprogress festgestellt, der einen Wechsel von Erst- zu Zweitlinienchemotherapie nach sich zieht, möglicherweise verbunden mit einem Wechsel von einem stationären zu einem ambulanten Behandlungsregime.

Viele Patienten und Angehörige tun sich schwer damit, hinzunehmen, dass eine Behandlungsstrategie, die sie anfänglich als erfolgreich empfunden haben, sich in eine erfolglose verwandelt, ohne dass sie selbst oder die behandelnden Ärzte ihr Handeln merklich verändert hätten. Nicht selten werden die Vergeblichkeitsbefürchtungen des Behandlungsbeginns und die stets hintergründige Angst vor einem unabwendbar schweren Sterben in der Folge revitalisiert. Der zeitgleiche Verlust der behütenden 
Tab. 1 Gemeinsame Arbeitsschritte beim Perspektivgespräch.

1. Die bisherigen Behandlungsschritte und ihre Begründung, die ehedem angestrebten Behandlungsziele sowie die tatsächlich erreichten Behandlungsziele werden chronologisch rekapituliert.

2. Die aktuellen Untersuchungsergebnisse werden gebündelt und in ihrer Kernbedeutung für den Krankheits- und Behandlungsverlauf erläutert.

3. Eine aktuelle Standortbestimmung hinsichtlich der Krankheit und ihrer Behandlung wird vorgenommen.

4. Die Ängste und Bedenken der Patienten und der Angehörigen werden erhoben.

5. Die Ängste und Bedenken werden inhaltlich auf ihren subjektiven Begründungskontext geprüft, Missverständnisse, Fehldeutungen, negativierende Vereinseitigungen und Zuspitzungen werden sichtbar gemacht und bearbeitet (gemeinsame Korrekturarbeit).

6. Eine Handlungsperspektive hinein in die nahe Behandlungszukunft wird erarbeitet. Die vorhandenen Handlungskompetenzen der Patienten und Angehörigen werden als integraler Bestandteil der Handlungsperspektive hervorgehoben (Förderung von Angst reduzierendem Handlungsfähigkeitserleben).

7. Angst vermindernde Behandlungsergänzungen werden erarbeitet und installiert.

Aspekte eines stationären Behandlungsregimes kann zudem aggravierend in diese Gemütslage hineinwirken.

Die Patienten und ihre Angehörigen wünschen sich in dieser Situation nach unserer Erfahrung ein Gespräch, das aus dem täglichen Arbeitsfluss erkennbar herausgeschnitten ist und ihrem Gefühl existenzieller Not gebührend Rechnung trägt. Sie erleben es als äußerst hilfreich, wenn dieses Gespräch klar erkennbare Konturen hat, an denen sie sich gut festhalten können. So fällt es ihnen leichter, ihre in Unordnung geratenen Gedanken und Gefühle zu sortieren. Sie suchen im Laufe des Gesprächs einen engen Kontakt zu den vertrauten Personen ihres Behandlungsalltags, um das Unterstützungsbündnis sichtbar und hörbar zu bekräftigen, das sie zu Behandlungsbeginn geschlossen haben.

Der behandelnde Stationsarzt lädt die Patienten und ihre Angehörigen zu einem Perspektivgespräch ein, in dem die bisherigen Kernschritte der Behandlung gemeinsam rekapituliert werden und eine Standortbestimmung der Krankheits- und Behandlungssituation vorgenommen wird. Unter Berücksichtigung der aktuellen Untersuchungsergebnisse wird die Behandlung gemeinsam neu ausgerichtet. Im Laufe dieses Prozesses werden 7 Arbeitsschritte unternommen ( Tab. 1). Dabei werden grundlegende Kommunikationsarbeiten geleistet in Richtung auf eine kongruentere und konsistentere Informationslage ( $\bullet$ Tab. 2).

\section{Das Konsensgespräch in der dissenten}

\section{Behandlungssituation}

\section{Bei dieser Gesprächsform sollten teilnehmen:}

Der Patient und ein Angehöriger seines Vertrauens (Vorsorgebevollmächtigter), der behandelnde Stationsarzt, die behandelnde Mitarbeiterin aus der Bezugspflege, die behandelnde Physiotherapeutin (optional), der Psychoonkologe (in Moderationsfunktion).

\section{Grundthema:}

- Die Sichtweisen der einzelnen Mitarbeiter des Behandlungsteams auf die Krankheits- und Behandlungssituation stehen sich widersprüchlich gegenüber. Es entstehen auseinanderstrebende Handlungstendenzen.

- Die Sichtweise der Mitarbeiter des Behandlungsteams auf die Krankheits- und Behandlungssituation ist in Widerspruch geraten zu der Sichtweise des Patienten und seiner Angehörigen. Sowohl die Patienten und ihre Angehörigen als auch die Mitarbeiter des Behandlungsteams leiden unter einer unentschieden-widersprüchlichen Kommunikation. Je nach Problemlage werden die an der Behandlung beteiligten Mitarbeiter zu einem grundlegenden Klärungsgespräch im Behandlungsteam eingeladen oder die Patienten und ihre Angehörigen zu einem grundlegenden Klärungsgespräch mit dem Behandlungsteam eingeladen.

Es soll erreicht werden, dass die Gesprächspartner gemeinsam in eine einvernehmliche Handlungsorientierung zurückfinden. Unter Moderation des Psychoonkologen werden 6 Arbeitsschritte vorgenommen ( Tab. 3).

\section{Das Konsensgespräch in der finalen Krankheitssituation}

\section{Bei dieser Gesprächsform sollten teilnehmen:}

der Patient und ein Angehöriger seines Vertrauens (Vorsorgebevollmächtigter), der behandelnde Stationsarzt, der Palliativmediziner, die behandelnde Mitarbeiterin der Bezugspflege, der Psychoonkologe, die zuständige Mitarbeiterin des Sozialdienstes (optional).

\section{Grundthema:}

Die Patienten, ihre Angehörigen und die behandelnden Stationsärzte befinden sich in einer medizinischen Unumkehrbarkeitssituation an der Schnittstelle zwischen tumororientierter palliativer Behandlung und palliativmedizinischer Symptombehandlung. Der behandelnde Arzt, der Palliativmediziner und der Psychoonkologe klären die Patienten und ihre Angehörigen darüber auf, dass die Grunderkrankung durch die tumororientierte Behandlung nicht mehr Entwicklung umkehrend behandelt wer-

Tab. 2 Grundlegende Kommunikationsarbeiten im Perspektivgespräch.

\section{Kongruenzarbeit}

- Die behandelnden Ärzte streben an, dass der Informationsstand der Patienten und ihrer Angehörigen und ihr eigener Informationsstand hinsichtlich der Krankheit und deren Behandlung möglichst deckungsgleich werden. Die Erwartungsangst der Patienten und ihrer Angehörigen, hinter den ärztlichen Mitteilungen könnte noch mehr Unheilvolles versteckt sein, soll sukzessive ausgeräumt werden.

- Die Patienten, ihre Angehörigen und die behandelnden Ärzte sollen immer öfter feststellen können, dass sie die grundlegenden Aspekte der Krankheits- und Behandlungssituation ähnlich sehen und von einem gemeinsamen Standpunkt, nebeneinander stehend, in die nahe Behandlungszukunft blicken (Angst reduzierendes Bündniserleben).

\section{Konsistenzarbeit}

- Die behandelnden Ärzte versuchen zusammen mit den Patienten und Angehörigen immer wieder die innere Behandlungslogik herauszuarbeiten. In psychisch und physisch belastenden Krankheits- und Behandlungssituationen fühlen Patienten und Angehörige sich sicherer, sofern sie sich an diesem Plausibilitätsstrang festhalten können.

- Selbst wenn die Behandlung vergeblich erscheint, wie zum Beispiel bei einem Tumorprogress unter chemotherapeutischer Behandlung, ängstigen sich Patienten und Angehörige deutlich weniger, sofern sie die Entscheidung für die Behandlung rückblickend als gut begründet und im Kern als richtig bewerten. 
Tab.3 Gemeinsame Arbeitsschritte beim Konsensgespräch in dissenten Behandlungssituationen.

1. Der Widerspruch wird im Einvernehmen formuliert (Problemformulierung).

2. Jeder Gesprächsteilnehmer begründet seine subjektive Sichtweise. Es werden nur Verständnisfragen bearbeitet, keine diskursiven Entgegnungen (Verständnissicherung).

3. Anhand der Dokumentation des Krankheits- und Behandlungsverlaufs wird die Datenlage im Hinblick auf den formulierten Widerspruch geprüft (Datenabgleich).

4. Die initiale Formulierung des Widerspruchs wird auf der Grundlage der aktuellen Informationslage neu bewertet und gegebenenfalls reformuliert (Problemreformulierung).

5. Die dissent gebliebenen Sichtweisen werden benannt und priorisierend gegeneinander abgewogen (Priorisierende Entscheidungsfindung).

6. Eine Handlungsvereinbarung wird formuliert und dokumentiert, die gemeinsam getragen wird (Konsente Handlungsvereinbarung).

den kann. Sie unterbreiten das Angebot einer palliativmedizinischen Symptombehandlung.

Die Patienten und Angehörigen erfahren, dass im Unterschied zur tumororientierten palliativen Behandlung, die auf die unmittelbare Beeinflussung der Grunderkrankung zentriert ist und dabei unabwendbare Beeinträchtigungen der Allgemeinverfassung des Patienten in Kauf nimmt, die palliativmedizinische Symptombehandlung auf eine unmittelbare Beeinflussung der Grunderkrankung verzichtet und sich gänzlich konzentriert auf die subjektiv spürbare Verbesserung der Allgemeinverfassung des Patienten, auf das Zurückgewinnen und Bewahren von Lebensqualität.

Nicht selten finden die Patienten - gerade nach einem langen, Kräfte zehrenden Behandlungsverlauf - eher in die palliativmedizinische Symptombehandlung als ihre Angehörigen, die noch sehr erfüllt sind von der Angst vor einer ungebremst voranschreitenden Tumorerkrankung. Ungewollt können Missstimmigkeiten aufkommen. Die Recherche nach möglicherweise übersehenen Behandlungsalternativen seitens der Angehörigen kann auf den Wunsch der Patienten treffen, es gut sein zu lassen, die bestehende Lebensqualität so lange zu bewahren, wie es eben geht. In dieser widersprüchlichen Situation wünschen sich die Patienten und ihre Angehörigen nach unserer Erfahrung ein aus der täglichen Kommunikation auf Station ausgegliedertes Konsensgespräch, das sie dabei unterstützt, ihre Hoffnungen hinsichtlich der Krankheit und ihrer Behandlung einvernehmlicher auszurichten, um mit dem kräftigen Gefühl emotionaler Geschlossenheit in die verbleibende gemeinsame Lebenszukunft zu gehen.

Typische Fragestellungen, die mit den Patienten und ihren Angehörigen im Konsensgespräch in der finalen Krankheitssituation bearbeitet werden, sind aufgeführt in $\bullet$ Tab. 4.

Im Anschluss an das Gespräch in der finalen Krankheitssituation wird den Patienten mit festem Blick auf die medizinische Indikation und ihre subjektive Bedürfnislage eine rehabilitativ ausgerichtete, palliativmedizinische Symptombehandlung auf Station angeboten (palliativmedizinische Komplexbehandlung), die das Erreichen ihrer Lebensziele möglich machen soll, zum Beispiel die Rückkehr nach Hause in ein möglichst privates Leben.
Tab. 4 Gemeinsam zu bearbeitende Fragestellungen beim Konsensgespräch in der finalen Krankheitssituation.

1. Welche Ängste und Bedenken haben der Patient und seine Angehörigen in der aktuellen Krankheits- und Behandlungssituation?

2. Welche - ihre Ängste und Bedenken vermindernden - Behandlungsziele wünschen der Patient und seine Angehörigen zu erreichen?

3. Welche Behandlungsziele kann die symptomatische Behandlung erfahrungsgemäß erreichen?

4. Welche Behandlungsziele werden gemeinsam angestrebt?

5. Welche Behandlungsschritte werden aus welchen Gründen gemeinsam unternommen, in welchem Zeitraum?

\section{Die palliativmedizinische Komplexbehandlung}

$\nabla$

Die Patienten und Angehörigen werden an der Schnittstelle von tumororientierter und palliativmedizinischer Symptombehandlung immer wieder von Unsicherheitsgefühlen und Erwartungsängsten geplagt, auch wenn über die gemeinsam vorausschauende Kommunikation bereits ein verlässlich vertrauensvolles Behandlungsbündnis entstanden ist. Sie versuchen nicht selten, ein wenig Sicherheitsgefühl zu bewahren, indem sie sich in ihrem unmittelbaren sozialen Umfeld an Gewohntem festhalten: Sie gehen in engeren Kontakt zu ihren Bezugspersonen in ihrem klinischen Lebensalltag, zu ihrem Arzt, zu ihrer Krankenschwester, zu ihrer Physiotherapeutin, zu ihren Mitpatienten in ihrem Krankenzimmer.

Eine palliativmedizinische Behandlung sollte diesem Bedürfnis entgegenkommen. Sie sollte nach Möglichkeit ohne einen einschneidenden Wechsel des behandelnden Personals oder des Behandlungsortes in Gang gesetzt werden als palliativmedizinische Komplexbehandlung, ausgeführt von einem konsiliarischen Palliativ-Care-Team. Wenn man so will, wird auf diese Weise eine ambulante Behandlungsform in die stationäre Behandlungsform integriert, der Funktionsbereich Palliativmedizin wird zu den bedürftigen Patienten getragen.

Idealerweise sollte ein konsiliarisches Palliativ-Care-Team bestehen aus:

einem Palliativmediziner, einem Lungenfacharzt, einem Arzt mit Zusatzausbildungen in Naturheilverfahren und Akupunktur, einer Krankenschwester mit Palliativ-Care-Ausbildung, einer Ausbildung in aktivierender, therapeutisch-begleitender Pflege, einer Ernährungsberaterin, einer Physiotherapeutin, die unter anderem spezialisiert ist auf Atemtherapie und Lungensport, einer Sozialarbeiterin, einem Psychoonkologen, einem Seelsorger und einer Sterbeamme.

Nach unseren Erfahrungen wäre es hilfreich, wenn die unterstützende Arbeit des Psychoonkologen, des Seelsorgers und der Sterbeamme über den Krankenhausaufenthalt der Patienten hinausreichen könnte. Sie sollten im Rahmen ihrer Dienstzeiten von außen telefonisch erreichbar sein über eine Unterstützungshotline. Es sollte ihnen möglich sein, Hausbesuche zu unternehmen. So könnten sie beispielsweise die Patienten und Angehörigen am Tag der Entlassung aus dem Krankenhaus nach Hause, in eine Pflegeeinrichtung oder ein Hospiz begleiten. Das potenziell Angst reduzierende, weil Sicherheit spendende Beziehungskontinuum mit den Patienten und Angehörigen könnte so weitestgehend erhalten bleiben.

Patienten, die im Krankenhaus sterben und ihre Abschied nehmenden Angehörigen benötigen eine wunschgerechte Begleitung. Dies sollte unseres Erachtens rund um die Uhr gewährleistet sein durch einen Seelsorger, eine Sterbeamme oder eine Krankenschwester mit Palliativ-Care-Ausbildung und einer Zusatz- 
ausbildung in Sterbebegleitung, die als verlässliche Bezugsperson im Krankenzimmer anwesend bleiben. Die Angehörigen verstorbener Patienten sollten sich, auch nachdem sie das Krankenhaus längst verlassen haben, in lastvollen Situationen der Trauer an die ihnen vertrauten Personen wenden können. Der Seelsorger und auch der Psychoonkologe sollten die Möglichkeit haben, sie in der ihnen gewohnten Weise, mitdenkend und mitfühlend zu unterstützen (Trauerbegleitung in Einzel- und Gruppengesprächen) [11].

\section{Fazit}

Die palliative Behandlung pneumologischer Erkrankungen bleibt aufgrund ihrer komplexen Anforderungen, gerade auch in Richtung auf eine bedürfnisgerechte Gestaltung, eine interdisziplinäre Herausforderung. Eine vorsorgend vorausschauende Kommunikation und eine Schutz und Aufgehobenheit bietende psychosoziale Patientenbegleitung sollten aufgrund der erwartbaren psychisch und physisch bedrängenden Symptomlagen einen steuernden Einfluss auf die medikamentöse und physikalische Therapie nehmen. Eine in diesem Sinne praxisgerechte kommunikative Weiterbildung der pneumologisch tätigen Ärzte erscheint wünschenswert.

\section{Interessenkonflikt}

$\nabla$

Die Autoren geben an, dass kein Interessenkonflikt besteht.

\section{Literatur}

1 Alberg AJ, Ford JG, Samet JM. Epidemiology of lung cancer: ACCP evidence-based clinical practice guidelines. (2nd edition). Chest 2007; 132: $29-55$

2 Dubey S, Powell CA. Update in lung cancer. Am J Respir Crit Care Med 2009; 179: 860

3 Bruera E, Neumann CM, Mazzocato C et al. Attitudes and beliefs of palliative care physicians regarding communication with terminally ill cancer patients. Palliat Med 2000; 14: 287-298

4 Temel JS, Greer JA, Muzikansky A et al. Early palliative care for patients with metastatic non-small-cell lung cancer. N Engl J Med 2010; 19: $733-742$

5 WHO Definition of Palliative Care. http://www.who.int/cancer/palliative/definition/en; Zugriff am 18.10.2010

6 Bausewein C, Fegg M, Radbruch L et al. Validation and application of the german version of the palliative outcome scale. J Pain Symptom Manage 2005; 30: 51 - 62

7 Enquete-Kommission Ethik und Recht der modernen Medizin des Deutschen Bundestags. 15. Wahlperiode. Zwischenbericht „Patientenverfügungen“. BT-Drs. 15/3700. Berlin: 2005

8 Goeckenjan G et al. Deutsche Gesellschaft für Pneumologie und Beatmungsmedizin und die Deutsche Krebsgesellschaft. Prävention, Diagnostik, Therapie und Nachsorge des Lungenkarzinoms. Interdisziplinäre S3-Leitlinie der Deutschen Gesellschaft für Pneumologie und Beatmungsmedizin und der Deutschen Krebsgesellschaft. Pneumologie 2010; 64 (Suppl. 2): e1 - e164. http://www.ncbi.nlm.nih.gov/pubmed/ 20217630

9 Radbruch L, Nauck F, Fuchs M et al. Working Group on the Palliative Care Units in Germany. What is palliative care in Germany? Results from a representative survey. J Pain Symptom Manage 2002; 23: 71 - 483

10 Fallowfield $L$. Truth sometimes hurts, but deceit hurts more. In: Surbone A, Zwitter M eds. Communication with the Cancer Patient. Information and Truth. Ann N Y Acad Sci 1997; 809: 525-537

11 Teno JM, Clarridge BR, Casey V et al. Family perspectives on end-of-life care at the last place of care. JAMA 2004; 291: 88-93 